\title{
Relaciones entre el autoconcepto y el rendimiento académico, en alumnos de Educación Primaria
}

\author{
Francisco Javier Peralta Sánchez \\ María Dolores Sánchez Roda
}

Orientadores de Centros de Secundaria

Almería, España

franperas@terra.es 


\section{RESUMEN}

Introducción. El autoconcepto como percepción que cada uno tiene de sí mismo es un componente del desarrollo de la personalidad. Los objetivos que nos proponemos se fundamentan en comprobar el grado de asociación y de predicción entre el autoconcepto y el rendimiento académico, así como determinar las propiedades psicométricas del cuestionario SDQ.

Método. La muestra estuvo formada por 245 alumnos de la Etapa de Educación Primaria de la provincia de Almería (España) que cursan sus estudios en centros docentes públicos y concertados. Obtuvimos datos relativos al autoconcepto de los mismos a través del la aplicación del cuestionario SDQ, así como de su rendimiento escolar a través de las calificaciones otorgadas por sus profesores. Tras contrastar la estructura factorial de la escala, establecimos los niveles de asociación y predicción, entre el autoconcepto y el rendimiento académico.

Resultados. Se obtiene una estructura factorial del cuestionario similar a la obtenida por Elexpru y unos índices de fiabilidad adecuados. Detectamos una estrecha relación entre el autoconcepto académico y las medidas de rendimiento académico. Además se ha constatado que el autoconcepto total y el autoconcepto académico son buenos predictores del rendimiento general.

Discusión. Nuestros resultados, en la edad de la muestra utilizada y en este estudio particular, apoyan la idea de que el autoconcepto total predice el rendimiento académico, dándose el hecho de que el autoconcepto no-académico predice de forma negativa el logro escolar (en lengua y matemáticas), mientras que el autoconcepto académico predice de forma potente y positiva tanto el logro general, como el de lengua y el de matemáticas. Consideramos que es necesario dar un tratamiento adecuado y suficiente al autoconcepto y a la autoestima y que se debe ofrecer al profesorado orientaciones metodológicas para trabajarlos a lo largo de todo el proceso educativo.

PALABRAS-CLAVE: autoconcepto escolar, rendimiento académico, asociación, predicción. 


\section{INTRODUCCIÓN}

El estudio del autoconcepto ha despertado un interés creciente en la investigación psicológica en los últimos años. A pesar de la profusión de trabajos encaminados a su estudio es difícil encontrar una definición unánime y aceptada del término autoconcepto, dado que su estudio se ha encarado desde diferentes perspectivas teóricas. Sin embargo, sí suele existir coincidencia entre los distintos autores en el carácter pluridimensional del término autoconcepto. Se considera que el autoconcepto está integrado por varias dimensiones, ámbitos o facetas, algunas de las cuales están más relacionadas con determinados aspectos de la personalidad (físicos, sociales, emocionales), mientras que otras aparecen más vinculadas al logro académico (en diferentes áreas y materias).

El autoconcepto «es el conjunto de percepciones o referencias que el sujeto tiene de sí mismo; (...) el conjunto de características, atributos, cualidades y deficiencias, capacidades y límites, valores y relaciones que el sujeto conoce como descriptivos de sí y que percibe como datos de su identidad» (Hamachek, 1981, citado por Machargo, 1991, p. 24). Por tanto, es el conjunto de conocimientos y actitudes que tenemos sobre nosotros mismos, las percepciones que el individuo se adjudica a sí mismo y las características o atributos que usamos para describirnos. Se entiende que es fundamentalmente una apreciación descriptiva y tiene un matiz cognitivo.

La importancia del autoconcepto radica en su notable contribución a la formación de la personalidad. La autoestima tiene que ver con la competencia social, ya que influye sobre la persona en cómo se siente, cómo piensa, cómo aprende, cómo se valora, cómo se relaciona con los demás y, en definitiva, cómo se comporta (Clark, Clemes y Bean, 2000; Clemes y Bean, 1996).

Por intentar llegar a una definición común del autoconcepto hemos optado por tomar como referencia el modelo teórico y la definición propuestos por Shavelson, Hubner y Stanton (1976). Estos autores definen el término autoconcepto como la percepción que cada uno tiene de símismo, que se forma a partir de las experiencias y las relaciones con el entorno, en las que las personas significativas desempeñan un papel importante.

El autoconcepto, como componente del desarrollo de la personalidad humana, tiene su propia naturaleza y peculiaridad. Varios autores (Shavelson et al.; 1976; citado por Garma y 
Elexpuru, 1999) han intentado precisar la naturaleza del término autoconcepto. En este sentido lo focalizan en relación a siete características o aspectos fundamentales: el autoconcepto constituye una dimensión psicológica; es multidimensional; tiene una organización jerárquica (un autoconcepto general y unos autoconceptos específicos); es estable, pero a medida que bajamos en la jerarquía, el autoconcepto se hace más específico y más susceptible de cambio; las distintas facetas del autoconcepto se van diferenciando entre sí con una mayor precisión, con la edad y la experiencia; el autoconcepto incluye tanto aspectos descriptivos como evaluativos; el autoconcepto puede diferenciarse de otros constructos con los que está relacionado, como por ejemplo, el rendimiento académico.

Algunos autores, como Harter (1986), realizan aportaciones interesantes, así el autoconcepto general o global va a estar determinado por el grado de importancia que demos a cada uno de los componentes específicos. Si al autodescribirnos nuestros juicios de valor son satisfactorios, entonces obtendremos un autoconcepto global positivo; en el caso contrario generaremos sentimientos negativos y por tanto repercutirá en un autoconcepto global negativo.

\section{Autoconcepto y rendimiento}

La Psicología de la Educación se viene preocupando de analizar distintos tipos de relaciones, tanto de asociación como de predicción, que existen entre autoconcepto y rendimiento académico (González-Pienda, Núñez, González-Pumariega, Álvarez, Roces, García, González, Cabanach, Valle, 2000; Núñez, González-Pienda; García, González-Pumariega, Roces, Álvarez, González, 1998). Sin embargo, a pesar de la profusión de los estudios, no existen estudios concluyentes sobre la naturaleza exacta de la dirección del vínculo que une a estas dos variables. En los resultados que se vienen obteniendo se pueden advertir distintas variables extrañas que pueden alterar en distinto grado los resultados obtenidos (Núñez y González-Pienda, 1994). Estos autores indican la necesidad de diferenciar cuatro posibles patrones o modelos de causalidad entre el autoconcepto y el rendimiento académico.

1) El rendimiento académico determina el autoconcepto. Las experiencias académicas de éxito o fracaso inciden significativamente sobre el autoconcepto y autoimagen del alumno más que lo contrario, lo cual podría ser explicado mediante el papel de las evaluaciones de los otros significativos, o de la teoría de la comparación social (Tajfel y Turner, 1986).De cara a la inter- 
vención psicopedagógica y puesto que la variable que influye es el rendimiento académico, lo prioritario que hay que modificar es el nivel de logro del alumnado, ya que éste contribuirá a cambiar el nivel de autoconcepto.

2) Los niveles de autoconcepto determinan el grado de logro académico. Para este modelo de relación causal también cabe poner en práctica importantes decisiones educativas. Puesto que el autoconcepto es el que determina los niveles de rendimiento académico y que, a su vez, el autoconcepto puede estar fuertemente influenciado por el tipo de contingencias que proporcionan otras personas significativas para el alumnado, entre las que no debemos despreciar las proporcionadas por el profesorado (principio de Pigmalión). Podemos inducir que sería posible incrementar los niveles de rendimiento escolar optimizando previamente los niveles de autoconcepto y muy específicamente los niveles de competencia percibida.

3) El tercer modelo de relación causal es el que postula que autoconcepto y rendimiento académico se influyen y determinan mutuamente.

4) Otros autores partidarios de este modelo postulan que la existencia de terceras variables pueden ser la causa tanto del autoconcepto como del rendimiento académico, entre las que podemos encontrar variables de tipo personal y ambiental, variables académicas o no académicas.

Además, se ha constatado los efectos beneficiosos que produce un buen nivel de autoconcepto. En estudios en los que se ha comparado a sujetos con un nivel de autoconcepto alto con otros sujetos con un nivel de autoconcepto bajo, se ha encontrado, a través de los informes de los profesores, que los primeros son considerados por el profesorado por ser más populares, cooperativos, persistentes en el trabajo de clase, por tener menores niveles de ansiedad, familias con mayor nivel de apoyo y expectativas de éxito futuro más altas (Hay, Ashman y Van-Kraayenoord 1998). 


\section{Objetivos de la investigación}

Esta investigación la hemos planteado teniendo en cuenta tres objetivos:

1. Determinar si la estructura factorial del cuestionario y otros índices psicométricos, con sujetos de sexto de educación primaria, es similar a la obtenida en otros estudios.

2. Comprobar el grado de asociación que puede haber entre el autoconcepto académico y el rendimiento académico general y específico de las áreas instrumentales.

3. Comprobar el grado de predicción que puede haber tomando las medidas de autoconcepto como factor fijo y las medidas de rendimiento académico como variable dependiente.

\section{Hipótesis}

Puesto que la muestra utilizada por nosotros difiere en edad de la utilizada por Elexpuru (1992), ya que nuestros alumnos cursan $6^{\circ}$ de educación primaria, nos planteamos la siguiente hipótesis:

$\mathrm{H}_{1} \quad$ La estructura factorial y los índices de fiabilidad están en consonancia con los hallados con muestras de alumnos de edades correspondientes a un curso académico inferior al que nosotros hemos empleado, demostrando el carácter multidimensional y jerárquico del constructo de autoconcepto.

Nos hemos planteado dos hipótesis de asociación:

$\mathrm{H}_{2} \quad$ El autoconcepto académico (TAC) estará más asociado con el rendimiento académico general (RACAGRAL), con el rendimiento académico en lengua (RACALEN) y con el rendimiento académico en matemáticas (RACAMAT) que el Autoconcepto NoAcadémico (TNAC) o que el Autoconcepto Total (TTOTAL).

$\mathrm{H}_{3} \quad$ Esperamos encontrar que determinados factores específicos de cada subescala se asocien más fuertemente con áreas de rendimiento escolar con las que tienen que ver directamente. Así, esperamos encontrar que el Factor 1 (Autoconcepto en matemáticas y asignaturas escolares en general -dimensión cognitiva-) tenga un alto grado de asociación con el rendimiento académico en matemáticas (RACAMAT) y con el rendimiento académico general (RACAGRAL). En cambio, esperamos encontrar que el Factor 3 (autoconcepto en lectura) esté más altamente asociado con el rendimiento académico en lengua (RACALEN).

$\mathrm{Y}$, finalmente, nos hemos planteado una hipótesis de predicción: 
$\mathrm{H}_{4} \quad$ El autoconcepto total (TTOTAL) debe predecir el rendimiento académico general (RACAGRAL), mientras que el total de autoconcepto no-académico (TNAC) debe predecir negativamente el rendimiento académico general (RACAGRAL).

\section{MÉTODO}

\section{Sujetos}

Participaron en el estudio un total de 245 alumnos, todos ellos de sexto curso de Educación Primaria. De este total, 122 fueron de género masculino y 123 de género femenino. El rango de edad de este alumnado oscilaba entre los 11 y los 13 años, con una media de la edad de 11.19 (desviación típica de .45). Todos ellos eran procedentes de cinco centros públicos de la comarca de la Alpujarra Almeriense (España) y un centro concertado de la misma zona.

\section{Materiales}

Como instrumentos de medida para esta investigación se han utilizado las medidas de autoconcepto y las medias de rendimiento académico.

1. Medida de autoconcepto. Se efectuaron a través de la escala SDQ de Marsh, Parker y Smith, a través de la adaptación española efectuada por Elexpuru (1992). Este cuestionario ha sido empleado para la evaluación del autoconcepto en multitud de ocasiones (Hay, 1997; Marsh y Seeshing, 1997; Marsh, 1992; Marsh, 1994; Pérez, Díaz, Núñez, y González-Pienda 1998; Plucker, Taylor, Callahan, y Tomchin 1997; Watkins y Akande 1992; Watkins y Mpofu 1994) tanto por los propios autores como por otras personas relevantes dentro del ámbito de la psicología.

El SDQ está compuesto por las subescalas de Autoconcepto Académico, Autoconcepto No-Académico y Autoconcepto Total de la escala.

1) La subescala de Autoconcepto Académico contiene los siguientes factores:

F1: Autoconcepto en matemáticas y asignaturas escolares en general (dimensión cognitiva).

F6: Autoconcepto en matemáticas y asignaturas escolares en general (dimensión afectiva).

F3: Autoconcepto en lectura.

F4: Autoconcepto en relaciones con los padres. 
Relaciones entre el autoconcepto y el rendimiento académico, en alumnos de Educación Primaria

Total de Autoconcepto Académico: la suma de las cuatro escalas antes mencionadas.

2) La subescala de Autoconcepto No-Académico contiene los siguientes factores:

F2: Autoconcepto en apariencia física.

F5: Autoconcepto en habilidad física y deportes.

F7: Autoconcepto en relaciones con los compañeros.

Total de la subescala Autoconcepto No-Académico (la suma de las tres escalas antes mencionadas).

3) La última subescala es la de Autoconcepto Total: la suma de las siete escalas o factores de autoconcepto.

Para cumplimentar este cuestionario, los sujetos deben responder a una serie de afirmaciones (items) a través de una escala de cinco intervalos, tipo lickert, que va desde totalmente falso a totalmente verdadero. Las puntuaciones en el SDQ están basadas en 72 items. El instrumento está diseñado para medir cuatro factores de autoconcepto no académico, tres factores de autoconcepto académico y tres medidas totales.

2. Medida del nivel de rendimiento escolar. Se ha efectuado a partir de las calificaciones asignadas por cada tutor a cada alumno, como una puntuación global respecto a su nota media. Las puntuaciones se adaptaron a una escala de 1 a 5 , en la que $1=$ suspenso, 2 = aprobado, $3=$ bien, 4 = notable y 5 = sobresaliente. Este mismo procedimiento para la obtención del Rendimiento Académico, al igual que en otros estudios (González-Pienda, Núñez y Valle 1992), se ha preferido realizar a través de las evaluaciones o de la nota media de las calificaciones realizadas por los propios profesores, en lugar de acudir a otras pruebas de rendimiento.

Existe evidencia empírica que apoya las evaluaciones de los profesores como un criterio suficientemente válido y fiable del rendimiento del alumno. Marsh, Parker y Barnes (1984); Marsh, Smith y Barnes (1985) han utilizado las evaluaciones de los profesores como criterio del rendimiento escolar, obteniendo altos coeficientes de consistencia entre distintas evaluaciones en diferentes momentos.

\section{Procedimiento}


La aplicación de las pruebas se ha realizado en las propias aulas de los alumnos, ya que en ningún caso los grupos sobrepasan en número los 25 alumnos y las aulas reúnen las condiciones idóneas para hacer una aplicación colectiva. La pasación de las pruebas estaba previamente concertada con el profesor-tutor.

El equipo de examinadores consta de una sola persona, con amplia experiencia en la aplicación de pruebas psicológicas (doce cursos como orientador escolar de zona -Equipos de Orientación Educativa- en los centros donde se ha realizado el estudio), por lo que ha existido una uniformidad de comportamiento para la aplicación de las pruebas en todas las aplicaciones.

La participación de los sujetos ha sido voluntaria, dándoseles la oportunidad de abandonar el aula si así lo deseaban antes de comenzar la aplicación de las pruebas. Los estudiantes completaron de forma individual y sin límite de tiempo el cuestionario. Siempre que el alumno pide aclaración sobre algún término se le proporciona.

El tratamiento estadístico se ha realizado mediante el paquete informático estadístico SPSS, versión 10.0, y el análisis de los datos que se exponen en esta investigación.

\section{RESULTADOS}

Los resultados que exponemos a continuación están basados en los tres objetivos que rigen esta investigación:

\section{Primer Objetivo:}

Determinar si la estructura factorial del cuestionario y otros índices psicométricos se asemejan a los obtenidos en otros estudios, empleando en este caso sujetos de sexto curso de educación primaria.

Elexpuru (1992, p.142) informa que obtuvo para alumnos de $5^{\circ}$ curso de E.G.B. un coeficiente alpha de .92. La fiabilidad de los totales académico y no-académico oscilaban entre .74 y 91. Otros autores, González-Pienda et al (2000), informan de la adecuada fiabilidad y validez de este instrumento de medida, así como una validez de constructo y predictiva del rendimiento académico satisfactorias. 
En la investigación nuestra hemos obtenido, para la escala total, un coeficiente alpha de ,8524. La fiabilidad de los totales no académico y académico son de ,8730 y de ,8075, respectivamente. En los factores los coeficiente alpha obtenidos oscilan entre ,6757 el más bajo y ,8790, para el más alto, con una media de 0,7773 .

En cuanto a la estructura factorial, con el método de componentes principales y rotación varimax, obligando a obtener 7 factores en la escala, es necesario señalar que los resultados obtenidos por nosotros evidencian una estructura muy parecida a la obtenida por Elexpuru (1992), en lo que a factores se refiere, coincidiendo también plenamente en los análisis de segundo orden, ya que obtenemos dos dimensiones (autoconcepto no-académico y autoconcepto académico). La medida de adecuación muestral de Kaiser-Meyer-Olkin fue de 0.794 y el test de esfericidad de Bartlett fue estadísticamente significativo $\left[\gamma^{2}(2556)=10400,853 ; \mathrm{p}=.0001\right]$. El porcentaje de varianza acumulado obtenido por los siete factores explican en total el 48,921\% de la varianza de los datos. Los porcentajes de varianza para los siete factores hallados oscilan entre $11,27 \%$ para el más alto y 3,59\% para el más bajo de ellos. Hemos considerado índices de saturación por encima de .35. En síntesis, estos resultados demuestran una vez más el carácter multidimensional y jerárquico del autoconcepto.

\section{Segundo Objetivo:}

Comprobar en qué medida el autoconcepto académico o áreas específicas del mismo están asociadas con el rendimiento académico general o con materias instrumentales (lengua o matemáticas).

En la segunda hipótesis planteábamos que el autoconcepto académico debe estar más asociado con el rendimiento académico general, con el rendimiento académico en lengua y con el rendimiento académico en matemáticas que el Autoconcepto No-Académico o que el Autoconcepto Total. 
Tabla 1.Coeficientes de correlación de Pearson entre las dimensiones y factores de la escala SDQ y las tres medias de rendimiento académico utilizadas (lengua, matemáticas y gral).

\begin{tabular}{|c|c|c|c|c|c|c|c|c|c|c|}
\hline Autoconceptos & RLen & Rmat & RGr & $\mathrm{F} 1$ & F3 & F4 & F6 & $\mathrm{F} 2$ & F5 & F7 \\
\hline $\begin{array}{l}\text { Total Acad. } \\
\text { (TAC) }\end{array}$ &, $37 * *$ &, $35^{* *}$ &, $35^{* *}$ &, $78 * *$ &, $70 * *$ &, $43 * *$ &, $84 * *$ &, $17 * *$ &, $15^{*}$ &, $31 * *$ \\
\hline F1: Mat.cogn. &, $41^{* *}$ &, $43 * *$ &, $42 * *$ & &, $30 * *$ & n. s. &, $72 * *$ &, $20 * *$ &, $15^{* *}$ &, $21 * *$ \\
\hline F3: lectura &, $25^{* *}$ &, $19 * *$ &, $23 * *$ &, $30 * *$ & &, $22 * *$ &, $30 * *$ & n. s. & n. s. &, $17 * *$ \\
\hline F4: rel. padres & n. s. & n. s. & n. s. & n. s. &, $22 * *$ & & n. s. &, $18^{* *}$ &, $20 * *$ &, $42 * *$ \\
\hline F6: mat. afec &, $24 * *$ &, $26 * *$ &, $22 * *$ &, $72 * *$ &, $35^{* *}$ &, $17 * *$ & & n. s. & n. s. &, $16^{* *}$ \\
\hline Total No-Acad. & n. s. &,$- 12 *$ & n. s. &, $22 * *$ & n. s. &, $29 * *$ &, $13 *$ &, $90 * *$ &, $83^{* *}$ &, $82 * *$ \\
\hline F2: apar. física &,$- 12^{*}$ &,$- 16^{*}$ &,$- 11^{*}$ &, $20 * *$ & n. s. &, $18 * *$ & n. s. & &, $60 * *$ &, $62 * *$ \\
\hline F5: HH fís dep. & n. s. & n. s. & n. s. &, $15^{*}$ & n. s. &, $20 * *$ & n. s. &, $60 * *$ & &, $56 * *$ \\
\hline F7: compañer. & n. s. & n. s. & n. s. &, $21 * *$ &, $17 * *$ &, $42 * *$ &, $16^{* *}$ &, $62 * *$ &, $56^{* *}$ & \\
\hline Autoc. Total &, $19 * *$ &, $17 * *$ &, $18 * *$ &, $67 * *$ &, $54 * *$ &, $47 * *$ &, $66^{* *}$ &, $63^{* *}$ &, $57 * *$ &, $68 * *$ \\
\hline \multicolumn{11}{|c|}{$* * \mathrm{p}<.01$} \\
\hline
\end{tabular}

Como podemos observar en la tabla anterior los resultados referentes a esta segunda hipótesis nos indican que, efectivamente, el autoconcepto académico (TAC) mantiene una estrecha, positiva y significativa asociación con las tres medidas de rendimiento académico utilizadas en este estudio, prácticamente con coeficientes de correlación muy similares. En cambio, la subescala de autoconcepto no-académico (TN-AC) no correlaciona significativamente con las medidas de rendimiento académico y, cuando lo hace, es de forma negativa y poco significativa, por ejemplo, entre autoconcepto no-académico y rendimiento académico en matemáticas.

La tercera de las hipótesis preveía encontrar que determinados factores específicos de cada subescala se asocien más fuertemente con áreas de rendimiento con las que tienen que ver directamente. Los análisis de asociación nos permiten afirmar que el factor 1 (autoconcepto en matemáticas y asignaturas escolares un general -dimensión cognitiva-) tiene un grado de asociación moderado, positivo y significativo, con las tres áreas de rendimiento, siendo algo superior en matemáticas. Respecto al factor 3 obtenemos coeficientes de correlación algo más bajos que en el caso anterior, pero en cualquier caso significativos y positivos, siendo superior la asociación obtenida en el área de lengua. El factor 4, aún perteneciendo a la escala académica, no correlaciona 
de forma significativa con ninguna de las tres medidas de rendimiento académico y sí lo hace en cambio con los tres factores que pertenecen a la escala no académica. El factor 6 (autoconcepto en matemáticas, dimensión afectiva) obtiene ligeramente un coeficiente algo más alto en el rendimiento de matemáticas que en el de lengua y el rendimiento en general.

También esperábamos que los factores de cada subescala correlacionaran con el resto de factores de su misma subescala más que con los de la otra subescala. Y así lo hemos encontrado, por ejemplo, el factor 1 arroja índices de correlación, desde moderados a altos, significativos y positivos con el resto de factores de su misma subescala y, con respecto a los tres factores de la escala no académica, obtiene coeficientes de correlación bajos, aunque significativos. El factor 3 solo correlaciona de forma significativa con los factores de su propia escala, pero no con los de la escala no académica. El factor 6 mantiene la línea que esperábamos de correlacionar más con los factores de su propia escala que con los de la otra. El factor 4 (autoconcepto en relación con los padres) tiene un comportamiento irregular ya que está más asociado con los factores de la escala no académica que con los de la suya propia.

En la subescala de autoconcepto no-académico, hemos hallado que los tres gfactores $(2,5$ y 7) obtienen coeficientes de correlación superiores (de moderados a altos) con respecto a los factores de su propia escala y coeficientes bajos con los de la escala académica.

\section{Tercer Objetivo:}

Comprobar en qué medida las dimensiones de la escala SDQ (autoconcepto académico y autoconcepto no-académico) y los siete factores son capaces de predecir tanto el rendimiento académico general, como el rendimiento académico en lengua y en matemáticas.

La cuarta de las hipótesis pronosticaba que el autoconcepto total (TTOTAL) debe predecir el rendimiento académico general (RACAGRAL), mientras que el total de autoconcepto noacadémico (TNAC) debe predecir negativamente el rendimiento académico general (RACAGRAL). Los resultados podemos observarlos en la tabla 2.

Tabla 2. Predicción de las tres medidas de rendimiento académico (gral, lengua y matemáticas) a partir 
Francisco Javier Peralta Sánchez y María Dolores Sánchez Roda

\begin{tabular}{|c|c|c|c|c|}
\hline \multicolumn{5}{|c|}{ de las dimensiones y factores de la escala $S D Q$} \\
\hline V. Predictora & V. Criterio & $\mathrm{R}^{2}$ & $\mathrm{~F}$ & Coef. Estandarizado \\
\hline Autoc. Total & & 0,034 & $(1,243)=8,599 * * *$ & $0,18(\mathrm{t}=2,932)^{* *}$ \\
\hline Autoc. Académ. & & & & $0,406(\mathrm{t}=6,726)^{* * * *}$ \\
\hline Autoc No-Acadé & & 0,161 & $(2,242)=24,45 * * * *$ & $-0,210(\mathrm{t}=-3,484) * * *$ \\
\hline $\begin{array}{l}\text { F1: AC matemá } \\
\text { cog }\end{array}$ & & & & $0,612(\mathrm{t}=7,442)^{* * * *}$ \\
\hline F2: Apar. física & & & & no significativo \\
\hline F3: AC lectura & & & & $0,138(\mathrm{t}=2,270) * * * *$ \\
\hline F4: AC padres & & & & $0,141(\mathrm{t}=2,251)^{*}$ \\
\hline F5: Hab. físicas dep & & & & no significativo \\
\hline F6: AC matem afec & & & & $-0,245(\mathrm{t}=-2,955) * * *$ \\
\hline $\begin{array}{l}\text { F7: AC compañe- } \\
\text { ros }\end{array}$ & Rend. Ac. Gral. & 0,279 & $(7,237)=13,10 * * * *$ & $-0,160(\mathrm{t}=-1,967)^{*}$ \\
\hline Autoc. Académ. & & & & $0,422(\mathrm{t}=7,022)^{* * * *}$ \\
\hline Autoc No-Acadé & & 0,179 & $(2,242)=26,372 * * * *$ & $-0,210(\mathrm{t}=-3,495)^{* * *}$ \\
\hline $\begin{array}{l}\text { F1: AC matemá } \\
\text { cog }\end{array}$ & & & & $0,571(\mathrm{t}=6,924)^{* * * *}$ \\
\hline F2: AC apa física & & & & $-0,149(\mathrm{t}=-1,920)^{*}$ \\
\hline F3: AC lectura & & & & $0,157(\mathrm{t}=2,575)^{* *}$ \\
\hline F4: AC padres & & & & $0,153(\mathrm{t}=2,448)^{* *}$ \\
\hline F5: Hab. físicas dep & & & & no significativo \\
\hline F6: AC matem afec & & & & $-0,211(\mathrm{t}=-2,531)^{* *}$ \\
\hline $\begin{array}{l}\text { F7: AC compañe- } \\
\text { ros }\end{array}$ & $\begin{array}{l}\text { Rend. Ac. Len- } \\
\text { gua }\end{array}$ & 0,274 & $(7,237)=12,793 * * * *$ & $-0,165(\mathrm{t}=-2,027)^{*}$ \\
\hline Autoc. Académ. & & & & $0,413(\mathrm{t}=6,869) * * * *$ \\
\hline Autoc No-Acadé & & 0,177 & $(2,242)=25,961 * * * *$ & $-0,227(\mathrm{t}=-3,771)^{* * * *}$ \\
\hline $\begin{array}{l}\text { F1: AC matemá } \\
\text { cog }\end{array}$ & & & & $0,598(\mathrm{t}=7,298 * * * *$ \\
\hline F2: AC apa física & Rend Ac. Matem. & 0,284 & $(7,237)=13,447 * * * *$ & $-0,235(\mathrm{t}=-3,251)^{* * * *}$ \\
\hline
\end{tabular}


Relaciones entre el autoconcepto y el rendimiento académico, en alumnos de Educación Primaria

\begin{tabular}{|l|l|l|l|l|}
\hline F4: AC padres & & & & $0,128\left(\mathrm{t}=2,056^{*}\right.$ \\
\hline \multirow{2}{*}{ F6: AC matem afec } & & & & $-0,177(\mathrm{t}=-2,144)^{*}$ \\
\hline \multirow{2}{*}{$\mathrm{p}<.05 * * \mathrm{p}<.01 * * * \mathrm{p}<.001 \quad * * * * \mathrm{p}<.0001$} & & \\
\hline
\end{tabular}

Respecto a la primera parte de esta hipótesis de predicción, lo que hemos hallado ha sido precisamente que el autoconcepto total y el autoconcepto académico son medidas que predicen el rendimiento académico general de un sujeto, mientras que el autoconcepto no académico predice de forma negativa el rendimiento académico general del sujeto. Esto es, a mejor autoconcepto no académico el modelo predice que los sujetos alcanzarán peor rendimiento académico general. Por tanto, estos resultados confirman las conjeturas que habíamos formulado respecto al poder predictivo del autoconcepto.

Además de los resultados buscados en nuestra hipótesis de tipo predictivo hemos encontrado otra serie de efectos estadísticos que presentamos a continuación. De este modo, encontramos varios efectos estadísticos significativos de tipo diferencial cuando tomamos cada uno de los factores de la escala SDQ con respecto al rendimiento académico general. El factor 1 (autoconcepto en matemáticas y asignaturas escolares en general -dimensión cognitiva-) actúa como una variable predictora de tipo positivo, respecto al rendimiento académico general. En el mismo sentido, el factor 3 (autoconcepto en lectura) también se comporta prediciendo de forma positiva y significativa el rendimiento académico general. El poder predictivo del factor 4 (autoconcepto en relación con los padres) es de tipo positivo, respecto al rendimiento académico general, pero con menor nivel de significatividad. Llama especialmente la atención el factor 6 (autoconcepto en matemáticas, dimensión afectiva), ya que muestra un comportamiento predictivo de tipo negativo respecto al rendimiento académico general, es decir, a mejor valoración que hacen los sujetos de su competencia en matemáticas peor rendimiento académico general obtienen. El factor 7 (autoconcepto en relación con los compañeros), al igual que el factor 6 , se muestra un comportamiento predictivo de tipo negativo, aunque poco significativo.

Con respecto a la predicción de las dimensiones y factores de la escala SDQ respecto al rendimiento académico en lengua podemos decir que la escala de autoconcepto académico y el factor 1 predicen de forma positiva y muy significativa el rendimiento académico en lengua. El 
mismo comportamiento muestran el factor 3 y el 4, pero con menores niveles de significatividad. El factor 6, que también pertenece a la escala académica, muestra un efecto predictivo de tipo negativo respecto al rendimiento en lengua.

La escala no académica del autoconcepto y los factores 2 y 7 que pertenecen a ella muestran un comportamiento predictivo de tipo negativo y muy significativo.

Finalmente, la predicción del rendimiento académico en matemáticas tiene también efectos diferenciales en función de si se trata de la escala académica y sus factores o de la no académica y los suyos los que utilizamos como variables criterio. La escala académica y el factor 1 tienen un poder predictor de tipo positivo y muy significativo del rendimiento que un sujeto puede alcanzar en matemáticas. El factor 4, también predice de forma positiva, aunque poco significativa el rendimiento en matemáticas y al factor 6 lo predice de forma negativa y poco significativa. La escala no académica de autoconcepto y el factor 2 que pertenece a ella, lo predicen de forma negativa y muy significativa.

\section{DISCUSIÓN}

Los objetivos de esta investigación consistían, en primer lugar, en determinar si la estructura factorial del cuestionario se asemeja a la obtenida, por ejemplo, por Elexpuru (1992), cuestión que hemos dejado aclarada satisfactoriamente, habiendo comprobado, a través del análisis factorial de primer orden, el carácter multidimensional de este constructo $\mathrm{y}$, a través del análisis factorial de segundo orden, su organización jerárquica. En segundo lugar, analizar el grado de asociación que existe entre el autoconcepto general o dimensiones específicas (autoconcepto académico y autoconcepto no-académico), así como los factores de la escala SDQ y el rendimiento académico, contemplado en tres aspectos: rendimiento académico general, rendimiento académico en lengua y rendimiento académico en matemáticas. Y en tercer lugar, en determinar el valor predictivo que puede poseer el autoconcepto, o dimensiones y factores específicos del mismo, respecto al rendimiento académico general o el rendimiento académico que puede alcanzar el alumnado en lengua y matemáticas. 
Para verificar el segundo objetivo planteamos la segunda y tercera hipótesis. En este sentido hemos visto como la dimensión académica del autoconcepto (autoconcepto académico) está asociado, con coeficientes de correlación cercanos a un nivel moderado, con las tres medidas de rendimiento académico utilizadas. También hemos comprobado que la escala de autoconcepto no académica no correlaciona con las medidas de logro escolar. Probablemente, si además de las medidas de logro escolar hubiéramos utilizado las medidas en otro tipo de actividades extraescolares o de ocio y tiempo, la asociación de la dimensión no académica del autoconcepto con ellas hubiera sido alta y positiva. Varios estudios recientes podrían confirmar este pronóstico (INCE, 2000a; 2001) donde se indica que el tipo de actividad extraescolar más realizado por alumnos de sexto de Educación Primaria en nuestro país, después de jugar en casa o con los amigos, es hacer deporte; actividad que realiza el 59\% de los sujetos. Este porcentaje de actividad es ligeramente superior (79\%) cuando la fuente de información proviene de los padres (INCE, 2000b).

La tercera de las hipótesis esperaba que factores específicos del autoconcepto (en matemáticas, en lectura) estuvieran más estrechamente asociados a áreas de aprendizaje específicas. Hemos hallado que el autoconcepto en matemáticas (dimensión cognitiva y la afectiva) tiene niveles de asociación muy parecidos con las tres medidas de logro. Los mismo podemos decir del autoconcepto en lectura. Parece, pues, que el autoconcepto que los sujetos tienen, ya sea en lectura o en matemáticas, se asocia en grado muy parecido al rendimiento académico que los sujetos obtienen en las áreas de lengua, matemáticas y general. Se observa que las medidas de autoconcepto con los padres no correlaciona con las áreas de logro académico. Probablemente porque la mayoría de los padres del alumnado de esta muestra tengan expectativas diferentes a los estudios de sus hijos o porque tengan un grado de insatisfacción considerable respecto a los resultados que van alcanzando sus hijos.

Nuestros resultados, respecto a los índices de correlación entre los factores de las dos dimensiones halladas, están en la línea de otros estudios (Elexpuru, 1992), lo que confirma que los factores específicos de cada una de las dimensiones se aglutinan entre sí, dándole consistencia a la dimensión a la que pertenecen. Destaca también el hecho de que el autoconcepto en habilidades físicas y deportes no correlaciona con el logro académico general. Nosotros interpretamos este hecho basándonos en que las disciplinas escolares, incluida la educación física, conllevan un componente académico, de dedicación, de esfuerzo (incluso intelectual) que no es el que desarro- 
llan los chicos y chicas en sus actividades de ocio y tiempo libre, salvo excepciones (federados, jugadores de clubs, etc).

Varios estudios avalan nuestros resultados. Alexander (1997) estudia la relación entre el rendimiento académico e inteligencia, estrategias de aprendizaje y rendimiento académico. Los datos de este estudio mostraron un alto grado de asociación positiva y significativa entre la autovaloración global y el autoconcepto académico, así como entre el rendimiento académico y el autoconcepto académico. Otro estudio similar al anterior nos informa que el autoconcepto académico se muestra favorablemente asociado con el rendimiento académico (Castor, 1997).

Un estudio realizado por Mboya (1998) ha evidenciado diferencias significativas en función de la edad del sujeto y el rendimiento académico en inglés, ciencias e historia, aunque no en matemáticas. Villarroel (2001) informa de varios resultados interesantes: (1) encontraron una asociación lineal entre autoconcepto y rendimiento académico; (2) también hallaron influencias recíprocas entre las expectativas de los profesores, el rendimiento académico de los estudiantes, y el autoconcepto de los estudiantes(3) efectos del rendimiento académico de los estudiantes en la percepción de los profesores.

El tercero de los objetivos (y cuarta de las hipótesis) estaba destinado a analizar el valor predictivo del autoconcepto respecto al rendimiento académico general. En la revisión realizada hemos encontrado estudios que analizan las relaciones existentes entre variables parecidas (Clemente, Albinana y Domenech, 1997): inteligencia, socialización, desadaptación escolar, autoconcepto y personalidad. También destacan los estudios de Acosta (2001) en los que, por ejemplo, al examinar la relación entre clima escolar, autoconcepto académico y rendimiento académico, afirma que los análisis de regresión múltiple arrojaban índices en el que las variables predictoras llegaban a explicar el 18\% de la varianza en el logro académico, aunque solamente la varianza explicada por el autoconcepto era estadísticamente significativa. Boulter (2002) utiliza el autoconcepto como predictor del ajuste o del rendimiento académico.

Nuestros resultados, en la edad de la muestra utilizada y en este estudio particular, apoyan la idea de que el autoconcepto total predice el rendimiento académico, dándose el hecho de que el autoconcepto no-académico predice de forma negativa el logro escolar (y el de lengua y matemá- 
ticas), mientras que el autoconcepto académico predice de forma potente y positiva tanto el logro general, como el de lengua y el de matemáticas. El valor predictivo de los factores 1 y 3 (matemáticas y lectura) nos hacen ver la importancia de percibirse competente en ellos de cara a mejorar en rendimiento académico, tanto general como el específico en lengua y matemáticas. El efecto estadístico hallado en el factor 6 (matemáticas, dimensión afectiva) tal vez influya en el hecho de que el alumnado tienda a sobrevalorar su propia capacidad y por eso se esfuercen en menor medida y los que más se infravaloran más se esfuercen.

El autoconcepto en relación con los compañeros (factor 7) se muestra como un predictor de tipo negativo del logro escolar general. Tal vez ya a partir de estas edades, el alumnado que se autopercibe con baja competencia académica intente compensar esos déficit a través de las relaciones sociales, entendidas muchas veces de forma errónea (reforzamiento no intencionado de sus propios compañeros y de los adultos), mediante conductas contrarias a la normas (Fernández, 2001; Kazdin y Buela-Casal, 2002).

Nuestros resultados hallados respecto al autoconcepto en relaciones con los padres, actuando como un predictor de tipo positivo del rendimiento académico general (así como el logro en lengua y el logro en matemáticas) nos da idea de la importancia del apoyo familiar y de otras variables psicosociales en el rendimiento académico de los hijos (Morvitz y Motta, 1992; Fantuzzo, Tighe y Childs 2000; Fantuzzo, Davis y Ginsburg 1995; Castejón, y Pérez 1998).

Respecto a la capacidad de predicción del autoconcepto (o sus dimensiones y factores) respecto del logro escolar específico en lengua y matemáticas hemos encontrado un comportamiento parecido. Esto es, por una parte la dimensión académica del autoconcepto (autoconcepto académico) y los factores en él incluidos tienen la capacidad de predecir el rendimiento académico tanto general, como el de lengua o el de matemáticas. Y por otro lado, la dimensión de autoconcepto no-académico y los factores a él asociados predicen de forma negativa el logro académico general como el de lengua y el de matemáticas.

Estos resultados están en consonancia con los obtenidos en otros estudios. Marsh (1990) encontró que las notas medias en los alumnos de 16-17 años estaban influidas de modo significativo por el autoconcepto académico medido el año anterior. La relación del autoconcepto académico y rendimiento se hace más fuerte con la edad, al menos en el período evolutivo estudiado 
por Marsh (1990). Sin embargo, es posible que el orden causal de estas variables pueda variar con la edad.

En la literatura encontramos estudios (Miujs, 1997) que analizan y encuentran relaciones de predicción significativas entre los constructos de autoconcepto y rendimiento académico. Nuestro estudio está en consonancia con otros que afirman que las variables afectivo y motivacionales son altamente influyentes en el rendimiento académico del alumnado, si cabe más que las propias aptitudes del sujeto (González-Pienda, Núñez, González-Pumariega, 2002). Este último estudio muestra un modelo unidireccional en el que la influencia del autoconcepto en el logro académico es estadísticamente significativo. Los resultados hallados por González-Pienda et al. (2002) pueden ser concordantes con otras investigaciones ( Shavelson y Bolus, 1982; Valle, Cabanach, Núñez y González-Pienda, 1998; Patrikakou, 1996). Como afirman González-Pienda et al. (2002) el tipo de estrategia utilizado para la recogida de datos, sea de tipo transversal o longitudinal, puede tener efectos condicionantes en los resultados obtenidos. De hecho, investigaciones que utilizan una estrategia longitudinal (Helmke y van Aken, 1995; Marsh, 1990; Marsh y Yeung, 1997; Marsh, Hau y Kong, 2002; Valentine, 2002) muestran la evidencia de relaciones recíprocas entre el autoconcepto y el logro académico.

González-Pienda et al. (2002) informan que, en la Conferencia Internacional sobre Motivación: $6^{\circ}$ taller de tareas de Motivación y Logro, celebrada en Grecia, presentaron los resultados de varias investigaciones (Núñez, González-Pienda, García, González-Pumariega, Roces, Cabanach, y Valle, 1998) en los que la relación entre autoconcepto y logro académico, medidos a través de una estrategia longitudinal (con un intervalo de un año entre el pretst y el postest), además de confirmar las relaciones recíprocas, los resultados hallados indican que el autoconcepto es la causa del logro académico inmediato, considerando que la influencia o relevancia del logro estarían en la base de una relación a largo plazo. En virtud de ello consideran al autoconcepto académico como una fuerza motivadora poderosa que responde al logro inmediato de los estudiantes. Sin embargo, este nivel de logro no afecta al autoconcepto de los estudiantes inmediatamente, sino que parece ser una fuente importante de información que repercute en el autoconcepto a largo plazo (un intervalo de 1 año en este estudio). Esto parece razonable del punto de vista de la estabilidad personal (González-Pienda, Núñez, González-Pumariega y García, 1997). 
Queda pendiente seguir investigando en la línea que hacen otros estudios (García, 1998; Fantuzzo, Tighe y Childs 2000; Fantuzzo, Davis y Ginsburg 1995; Castejón y Pérez 1998; Morvitz y Motta. 1992) de modo que podamos obtener más información sobre determinadas variables psicosociales y de tipo familiar (número de hermanos, lugar de nacimiento entre los hermanos, profesión de los padres, pautas educativas de los padres, factores condicionantes del contexto social o del barrio donde vive el alumno, etc) y la generación de un autoconcepto adaptado y/o desadaptado. El propósito debe servir para elaborar una guía a través de la cual podamos hacer propuestas de intervención y entrenamiento dirigidas a padres y/o docentes y optimizar los procesos educativos.

\section{CONCLUSIONES}

Es cierto que la formación del autoconcepto, principalmente el de tipo académico, no es sólo tarea del profesor-tutor, sino que intervienen el resto de profesionales que dentro de los centros educativos desarrollan su labor. Por ello seguimos reivindicando la necesaria formación que los docentes, al margen de la especialidad docente que impartan, deben tener una necesaria formación en temas comunes que competen, como agentes educativos, al equipo docente de cada uno de los alumnos. Por ello, desde cada centro educativo, la formación y perfeccionamiento en temas de competencia y desarrollo personal y social del alumno: autoconcepto, autoestima, habilidades sociales, desarrollo personal, mediación escolar, convivencia, resolución de conflictos, etc, (Haeusler y Milicic, 1996; Machargo, 1991; Trianes, 1996; De la Fuente, 1999; Merrell y Gimpel, 1998; Trianes, Muñoz y Jiménez, 1997; Trianes, De la Morena y Muñoz, 1999; Fernández, 2001; Torrego, 2000; Merrell, Streeter, Boelter, Caldarella y Gentry, 2001, Monjas y González, 2000) deben ser abordados a través de los planes de perfeccionamiento del profesorado de cada centro educativo.

En definitiva, consideramos que es necesario dar un tratamiento adecuado y suficiente al autoconcepto y a la autoestima (Carr, Kurtz-Costes, 1994; Gil, 1998; Machargo, Alonso, Quintana, Rojas y Santana, 1996), y que se debe ofrecer al profesorado orientaciones metodológicas para trabajarlos a lo largo de todo el proceso educativo, de modo que este tipo de intervención psicoeducativa pueda servir de camino para mejorar el rendimiento académico (Castejón, Navas y Sampascual, 1996; González, 1999). 


\section{REFERENCIAS}

Acosta, E. S. (2001). The relationship between school climate, academic self-concept, and academic achievement. Dissertation Abstracts International Section A: Humanities and Social Sciences, 62 (5-A): 1717

Alexander, S. (1997). The relationship of self-concept, IQ, academic performance, and stressors to coping abilities for urban African-American gifted students. Dissertation Abstracts International Section A: Humanities and Social Sciences, 58 (5-A): 1575.

Boulter, L. T. (2002). Self-concept as a predictor of college freshman academic adjustment. College Student Journal. 36 (2), 234-246

Carr, M., y Kurtz-Costes, B. (1994). Is being smart everything? The influence of student achievement on teacher's perceptions. British Journal of Educational Psychology, 64, 263-276.

Castejón, J. L. y Pérez, A. M. (1998). Un modelo causal-explicativo sobre la influencia de las variables psicosociales en el rendimiento académico A causal-explicative model about the influence of psycho-social variables on academic achievement. Bordón, 50, 171-185.

Castejón, J. L., Navas, L. y Sampascual, G. (1996). Un modelo estructural del rendimiento académico en matemáticas en la educación secundaria. Revista de Psicología General y Aplicada, 49 (1), 27-43.

Castor, S. E. (1997). Locus of control and self-concept in achieving and underachieving gifted students. Dissertation Abstracts International: Section B: The Sciences and Engineering. 57 (10-B): p. 6603

Clark, A., Clemes, H. y Bean, R. (2000). Cómo desarrollar la autoestima en adolescentes. Madrid: Editorial Debate

Clemente, A, Albinana, P y Domenech, F. (1997). Predicción del rendimiento académico en estudiantes de Secundaria a través de los factores de Inteligencia, Socialización, Desadaptación Escolar, Autoconcepto y Personalidad. / Prediction of academic performance in secondary students using factors of intelligence, socialization, school maladjustment, self-concept, and personality. Revista de Psicología de la Educación, 22, 55-68

Clemes, H. y Bean, R. (1996). Cómo desarrollar la autoestima en los niños. Madrid: Editorial Debate. 
De la Fuente, J. (1999). Educación para la construcción personal. Bilbao: Desclée de Brouwer. Elexpuru, I. (1992). El autoconcepto en los alumnos de 8 a 11 años de edad a través del SDQ. En A. Villa (Coord), Autoconcepto y educación: teoría, medida y práctica pedagógica (pp. 123-189). Vitoria: Servicio Central de Publicaciones del Gobierno Vasco.

Fantuzzo, J. W., Davis, G. Y. y Ginsburg, M. D. (1995). Effects of parental involvement in isolation or in combination with peer tutoring on student self-concept and mathematics achievement. Journal of Educational Psychology, 87, 272-281.

Fantuzzo, J. W., Tighe, E. y Childs, S. (2000). Family involvement questionnaire: A multivariate assessment of family participation in early childhood education. Journal of Educational Psychology, 92, 367-376.

Fernández, I. (Coord.) (2001). Guía para la convivencia en el aula. Barcelona: Ciss-Praxis.

García, F. J. (1998). Aproximación conceptual a las relaciones escuela-familia A conceptual approach to the relations between school and family . Bordón, 50, 23-34.

Garma, A. M. y Elexpuru, I. (1999). El autoconcepto en el aula. Barcelona: Edebé.

Gil, R. (1998). Valores humanos y desarrollo personal: tutorías de educación secundaria y escuelas de padres. Madrid: Escuela Española.

González, E. (1999). Necesidades educativas especiales: intervención psicoeducativa. Madrid: CCS.

González-Pienda, J. A., Núñez, J. C., González-Pumariega, S. y García, M. (1997). Self-concept, self-esteem and school learning. Psicothema, 9, 271-289.

González-Pienda, J. A., Núñez, J. C. y González-Pumariega, S. (2002). A structural equation model of parental involvement, motivational and aptitudinal characteristics, and academic achievement. The Journal of Experimental Education. 70 (3): 257-287

González-Pienda, J. A., Núñez, J. C., Valle, A. (1992). Procesos de Comparación Externa/interna, Autoconcepto y Rendimiento Académico. Rev. de Psicología General y Aplicada, 45 (1), 73-81.

González-Pienda, J. A., Núñez, J. C., González-Pumariega, S., Álvarez, L., Roces, C., García, M., González, P., Cabanach, R. G., Valle, A., (2000). Autoconcepto, proceso de atribución causal y metas académicas en niños con y sin dificultades de aprendizaje. Psicothema, 12 (4), 548-556.

Haeussler, P., y Milicic, N. (1996). Confiar en uno mismo. Programa de autoestima. Madrid: CEPE. 
Hamachek, D. E. (1981). Encuentros con el yo. México: Interamericana.

Harter, S. (1986). Processes Underlying Childrens Self-Concept; en J. Suls (ed.). Psicological Perspectives en the Self. (Vol. III). Hillsdale, New Jersey: Lawrence Erlbaun Associates.

Hay, I. (1997). Investigating the influence of achievement on self-concept and intra-class design and comparison of the PASS and SDQ Self-Concept Test. British Journal of Educational Psychology, 67, 311-321.

Hay, I., Ashman, A. F., Van-Kraayenoord, C. E. (1998). Educational characteristics of students with high or low self-concept. Psychology in the Schools. 35 (4): 391-400

Helmke, A. y Van Aken, M. A. G. (1995). The causal ordering of academic achievement and self-concept of ability during elementary school: A longitudinal study. Journal of Educational Psychology, 87, 624-637.

Instituto Nacional de Calidad y Evaluación, INCE (2000a). Perfil del alumnado sexto curso de Educación Primaria. Resumen informativo, 1, Julio, 1-6. Recuperado en julio de 2001 de http://www.ince.mec.es

Instituto Nacional de Calidad y Evaluación, INCE (2000b). Las familias de los alumnos de sexto curso de Educación Primaria. Resumen informativo, 2, Julio, 1-6. Recuperado en julio de 2001 de http://www.ince.mec.es

Instituto Nacional de Calidad y Evaluación, INCE (2001). Actividades de los alumnos fuera del horario escolar 2. Actividades extraescolares. Resumen informativo, 11, Mayo, 1-6. Recuperado en julio de $2001 \mathrm{de} \mathrm{http://www.ince.mec.es}$

Kazdin, A. E. y Buela-casal, G. (2002). Conducta antisocial. Evaluación, tratamiento y prevención en la infancia y la adolescencia. Madrid: Pirámide.

Machargo, J. (1991). El profesor y el autoconcepto de sus alumnos. Teoría y práctica. Madrid: Escuela Española

Machargo, J., Alonso, E., Quintana, R., Rojas, M. y Santana, A. (1996). Programa de actividades para el desarrollo de la autoestima. Madrid: Escuela Española.

Marsh, H. W. (1990). Causal ordering of academic self-concept and academic achievement: A multivariate, longitudinal panel analysis. Journal of Educational Psychology, 82, 646-656. 
Relaciones entre el autoconcepto y el rendimiento académico, en alumnos de Educación Primaria

Marsh, H. W. (1992). The Self-Description Questionnaire (SDQ) II. A Theoretical and empirical basis for measurement of multiple dimensions of adolescents' self-concept: An interim test manual and a research monograph. Macarthur, Australia: University of Western Sydney.

Marsh, H. W. (1994). Using the national longitudinal study of 1988 to evaluate theoretical models of self-concept: The Self-Description Questionnaire. Journal of Educational Psychology, 86, 439-456.

Marsh, H., y Seeshing, A. (1997). Causal effects of academic self-concept on academic achievement: structural equation of longitudinal data. Journal of Educational Psychology, 89 (1), 41-54.

Marsh, H. W., y Yeung, A. S. (1997). Causal effects of academic self-concept on academic achievement: Structural equation models of longitudinal data. Journal of Educational Psychology, 89, 41-54.

Marsh, H. W., Hau, K. y Kong, C. (2002). Multilevel Causal Ordering of Academic Self-Concept and Achievement: Influence of Language of Instruction (English Compared With Chinese) for Hong Kong Students. American Educational Research Journal, 39 (3): 727-63.

Marsh, H. W., Parker, J. W. y Barnes, J. (1984). Multidimensional adolescent self-concept: Their relationship to age, sex, and academic measures. American Educational Research Journal, 22, 422-444.

Marsh, H. W., Smith, I. D. y Barnes, J. (1985). Multidimensional self-concept: Relationship with sex and academic achievement. Journal of Educational Psychology, 77, 581-596.

Mboya, M. M. (1998). Self-concept of academic ability as a function of sex, age, and academic achievement among African adolescents. Perceptual and Motor Skill, 87 (1): 155-161.

Merrell, K. W. y Gimpel, G. A. (1998). Social skills of children and adolescents: Conceptualization, assessment, treatment. Mahwah, NJ: Laurence Erlbaum Associates.

Merrell, K. W, Streeter, A. L., Boelter, E. W., Caldarella, P. y Gentry, A. (2001). Validity of the Home and Community Social Behavior Scales: Comparisons with five behavior-rating scales. Psychology in the Schools. 38 (4), 313-325.

Miujs, D. (1997). Symposium: self perception and performance. Predictors of academic self-concept a longitudinal perspective. British Journal of Educational Psychology, 67, 263-277.

Monjas, Ma I. y González, B. P. (Dir) (2000). Las habilidades sociales en el currículum. Madrid: Ministerio de Educación, Cultura y Deporte (CIDE). 
Morvitz, E., y Motta., R. W. (1992). Predictors of self-esteem: The roles of parent-child perceptions, achievement, and class placement. Journal of Learning Disabilities, 25, 72-80.

Núñez, J. C. y González-Pienda, J.A. (1994). Determinantes del rendimiento académico. Oviedo. Servicio de Publicaciones de la Universidad.

Núñez, J. C., González-Pienda, J. A., García, M., González-Pumariega, S., Roces, C., Cabanach, R. y Valle, A. (1998). Causal relationship between the self-concept and academic achievement. International Conference on Motivation: 6th Workshop on Achievement and Task Motivation, Thessaloniki, Greece.

Núñez, J. C., González-Pienda; J. A., García, M., González-Pumariega, S., Roces, C., Álvarez, L., González, Ma . C. (1998). Estrategias de aprendizaje, autoconcepto y rendimiento académico. Psicothema, 10 (1), 97-109.

Patrikakou, E. N. (1996). Investigating the academic achievement of adolescents with learning disabilities: A structural modeling approach. Journal of Educational Psychology, 88, 435-450.

Pérez, M. V., Díaz, A., Núñez, J. C., y González-pienda, J. A. (1998). Adaptación del Self-Description Questionnaire (SDQ-II) en Chile. Aportes para su validez transcultural Adaptation of the Self-Description Questionnaire (SDQ II) in Chile. Contributions for its cross-cultural validity . II Congreso Iberoamericano de Psicología. Madrid: COP/SIP.

Plucker, J., Taylor, J. W., Callahan, C., y Tomchin, E. (1997). Mirror, mirror, on the wall: Reliability and validity evidence for the Self-Description Questionnaire-II with gifted students. Educational and Psychological Measurement, 57, 704-713.

Shavelson, R. J., Hubner, J. J. y Stanton, J.C. (1976). Self Concept: Validation of Construct Interpretations. Review of Educational Research, 46, 407-441.

Shavelson, R. J., y Bolus, R. (1982). Self-concept: The interplay of theory and method. Journal of Educational Psychology, 74, 3-17.

Tajfel, H. y Turner, J. C. (1986). The social identity theory of intergroup behavior. En S. Worchel y W. Austin (Eds), Psychology of intergroup relations. Chicago: Nelson-Hall.

Torrego, J. C. (Coord.) (2000). Mediación de conflictos en instituciones educativas. Manual para la formación de mediadores. Madrid: Narcea.

Trianes, $\mathrm{M}^{\mathrm{a}}$ V. (1996). Educación y competencia social. Un programa en el aula. Archidona: Aljibe. 
Trianes, Ma . V., Muñoz, A. y Jiménez, M. (1997). Competencia social, su educación y tratamiento. Madrid: Pirámide, Colección Ojos Solares.

Trianes, M. V.; de la Morena, M. L. y Muñoz, A. (1999). Relaciones sociales y prevención de la inadaptación social y escolar. Málaga: Aljibe.

Valentine, J. C. (2002). The relation between self-concept and achievement: A meta-analytic review. Dissertation Abstracts International: Section B: The Sciences and Engineering, 62 (9-B): p. 4278

Valle, A., Cabanach, R., Núñez, J. C. y González-Pienda, J. A. (1998). Cognitive-motivational variables, approaches to learning, and academic achievement. Psicothema, 10, 393-412.

Villarroel, V. A. (2001). Relación entre autoconcepto y rendimiento académico. Psykhe: Revista de la Escuela de Psicología. 10 (1): 3-18.

Watkins, D. y Akande, A. (1992). The internal structure of the Self-Description Questionnaire: A Nigerian investigation. British Journal of Educational Psychology, 62, 120-125.

Watkins, D. y Mpofu, E. (1994). Some Zimbabwean evidence of the internal structure of the Self-Description Questionnaire-I. Educational and Psychological Measurement, 54, 967-972. 